\title{
Generalization of Ryan's theorem: Probing tidal coupling with gravitational waves from nearly circular, nearly equatorial, extreme-mass-ratio inspirals
}

\author{
Chao $\mathrm{Li}^{1}$ and Geoffrey Lovelace ${ }^{2}$ \\ ${ }^{1}$ Theoretical Astrophysics, California Institute of Technology, Pasadena, California 91125, USA \\ ${ }^{2}$ Center for Radiophysics and Space Research, Cornell University, Ithaca, New York 14853, USA \\ (Received 28 February 2007; published 19 March 2008)
}

\begin{abstract}
Extreme-mass-ratio inspirals (EMRIs) and intermediate-mass-ratio inspirals (IMRIs) - binaries in which a stellar-mass object spirals into a massive black hole or other massive, compact body-are important sources of gravitational waves for LISA and LIGO, respectively. Thorne has speculated that the waves from EMRIs and IMRIs encode, in principle, all the details of (i) the central body's spacetime geometry (metric), (ii) the tidal coupling (energy and angular momentum exchange) between the central body and orbiting object, and (iii) the evolving orbital elements. Fintan Ryan has given a first partial proof that this speculation is correct: Restricting himself to nearly circular, nearly equatorial orbits and ignoring tidal coupling, Ryan proved that the central body's metric is encoded in the waves. In this paper we generalize Ryan's theorem. Retaining Ryan's restriction to nearly circular and nearly equatorial orbits, and dropping the assumption of no tidal coupling, we prove that Thorne's conjecture is nearly fully correct: the waves encode not only the central body's metric but also the evolving orbital elements and (in a sense slightly different from Thorne's conjecture) the evolving tidal coupling.
\end{abstract}

DOI: $10.1103 /$ PhysRevD.77.064022

PACS numbers: 04.30.Db, 04.70.- s, 04.80.Nn

\section{INTRODUCTION AND SUMMARY}

The LIGO-GEO-VIRGO-TAMA network of broadband ground-based laser interferometers, aimed at detecting gravitational waves in the high-frequency band 10$10^{4} \mathrm{~Hz}$, is already operating at or near its initial design sensitivities. In the next decade, LISA (the Laser Interferometer Space Antenna) will open up the low-frequency gravitational-wave window $\left(10^{-4}-0.1 \mathrm{~Hz}\right)$.

Among the most important sources of gravitational waves for LISA are extreme-mass-ratio inspirals (EMRIs), which are systems in which a small object (with mass $\mu \sim M_{\odot}$ ) orbits a supermassive black hole or other central body (boson star [1,2] or soliton star [3] or naked singularity or ...) with mass $M \sim 10^{6} M_{\odot}$. Recently, Brown and collaborators [4] and Mandel, Brown, Gair, and Miller [5] estimated ${ }^{1}$ that advanced detectors in LIGO (the Laser Interferometric Gravitational Wave Observatory) could detect up to $\sim 1-30 \mathrm{yr}^{-1}$ intermediate mass ratio inspirals (IMRIs), which are analogous to EMRIs but have less massive central bodies (masses $M$ in the range of $\sim 10^{2}-10^{4} M_{\odot}$ ).

\footnotetext{
${ }^{1}$ Event-rate estimates for IMRIs are very uncertain, since the abundance (and even existence of) intermediate-mass black holes - the typical central bodies in IMRIs - presently is not well understood. A discussion of intermediate-mass black-hole abundances is given in [5].
}

Thorne has conjectured ${ }^{2}$ that the waves from an EMRI or IMRI contain, encoded in themselves (at least in principle): (i) the spacetime geometry (metric) of the massive central body, (ii) the tidal coupling (evolving rate of energy and angular momentum exchange) between the orbiting object and the central body, and (iii) the evolving orbital elements. This conjecture (which has been partially proved; see below) has motivated placing EMRIs high on LISA's list of target sources [9], and has motivated research to: (a) prove Thorne's conjecture with the widest generality possible, or, if it is false, determine what information actually is encoded in the EMRI and IMRI waves [4,10]; (b) develop data analysis techniques for searching for EMRI and IMRI waves in LISA [11,12] and LIGO [13] data; (c) scope out the accuracy with which LISA and LIGO can extract the encoded information from EMRI

\footnotetext{
${ }^{2}$ Thorne's conjecture has grown over time. Originally, in the early 1990s, he conjectured (or, more precisely, asserted!) that the waves encode "a portion" of the spacetime geometry (e.g., p. 326 of [6]). By 1994, when Fintan Ryan proved his theorem, Thorne was arguing that the entire spacetime geometry would be encoded (see, e.g., the introduction to Ryan's paper [7]). In 2002, when thinking about how LISA might test the laws of black-hole physics, Thorne realized that the tidal coupling might also be encoded along with the central body's spacetime geometry; see [8]. Only recently, when advising the authors about their research, did Thorne realize that the evolving orbital elements might also be extractable (private communication).
} 
and IMRI waves (and if the central body appears to be a black hole, the accuracy with which its properties agree with those of a hole) [14,15]; and (d) develop data analysis techniques for extracting the waves' information [16].

Fintan Ryan [7] has proved a theorem that is an important step toward verifying Thorne's conjecture. Specifically, he has proved that it is possible in principle to recover the full spacetime geometry from EMRI waves under the following assumptions: (i) the central body is general-relativistic, stationary, axisymmetric, reflectionsymmetric, and asymptotically-flat (SARSAF), (ii) the small object travels on a nearly circular and nearly equatorial orbit, and (iii) there is no tidal coupling. Moreover, Ryan has shown that the multipole moments that determine the spacetime geometry are redundantly encoded in the gravitational waves and can be extracted using either of the two precession frequencies (about a circular orbit and about the equatorial plane) or the waves' phase evolution.

The purpose of this paper is to generalize Ryan's theorem. We retain assumptions (i) and (ii) (SARSAF spacetime and nearly circular, nearly equatorial orbit) but relax assumption (iii) by allowing for a small amount of tidal coupling. We show that in this case, Thorne's conjecture is nearly correct: the waves encode not only the central body's metric but also the evolving orbital elements and (in a sense slightly different from Thorne's conjecture) the evolving tidal coupling. (Assumption (ii), that the orbit is nearly circular and nearly equatorial, is relaxed in a companion paper by Li [10]. Assumption (i) has been generalized to the case of electrovacuum spacetimes in [17].)

Motivated by the result of Fang and Lovelace [18] that the only unambiguous part of the tidal coupling is the timedependent, dissipative portion (at least when the central body is a nonspinning black hole and the orbit is large and circular), we characterize the tidal coupling by the rates of energy and angular momentum exchange between the central body and the orbiting object, $\dot{E}_{\text {body }}$ and $\dot{L}_{\text {body }}$. (Throughout this paper, a dot means derivative with respect to the coordinate time $t$, which is the time measured by an inertial observer in the asymptotically flat region of the spacetime.) Actually, we only need to consider $\dot{E}_{\text {body }}$, because once it is known, $\dot{L}_{\text {body }}$ can be deduced from the standard energy-angular momentum relation for circular orbits and their influence on waves and tides, $\dot{E}=\Omega_{\text {orbit }} \dot{L}$. (Here $\Omega_{\text {orbit }}$ is the orbital angular velocity, which is the same as the waves' observed primary angular frequency aside from a factor 2.)

This paper is organized as follows: In Sec. II, we begin by noting that, when there is a small amount of tidal coupling (as we assume), then the redundancy in Ryan's analysis is broken. One can still use Ryan's algorithm for the precession frequencies to recover the central body's spacetime geometry. Then, by making use of the observed (time-independent) spacetime geometry and the measured, evolving amplitudes associated with the precession fre- quencies, one can also recover from the EMRI waves the evolving orbital parameters. Having relied on nondissipative aspects of the waves to deduce the spacetime geometry and orbit, one can then - as we show in Sec. III-use the waves' dissipation-induced phase evolution to deduce the tidal coupling.

In our somewhat delicate discussion of deducing the tidal coupling (Sec. III), we begin by noting that the sum of the power radiated to infinity and the power fed into the central body via tidal coupling, $\dot{E}_{\text {total }}=\dot{E}_{\infty}+\dot{E}_{\text {body }}$ is equal to the power lost from the orbit, which can be deduced from the waves' observed phase evolution. We assume that the energy absorbed into the inspiraling object is negligible. This is justified so long as the frequencies of all the object's normal modes are far higher than the orbital frequency. This is guaranteed to be true if the object is a black hole, since its normal-mode frequencies are greater than or of order the orbital frequency for a particle traveling around it, which in turn is far higher than its own orbital frequency around the central body. For a white dwarf inspiraling into a massive central body (LISA EMRI), this assumption may fail [19], and for a neutron star inspiraling into an intermediate-mass black hole (LIGO IMRI) it might fail, due to the star's inertial modes being tidally excited [20].

The central body influences this observed $\dot{E}_{\text {total }}$ in two ways: (i) by generating a nonzero $\dot{E}_{\text {body }}$, the quantity that interests us, and (ii) by very slightly altering $\dot{E}_{\infty}$. To help quantify these two body influences, in Sec. III B we show how one can deduce, from the observations, the rate $\dot{E}_{\infty \mathrm{NBI}}$ that energy would be radiated to infinity if there were no body influences. The difference between the measured $\dot{E}_{\text {total }}$ and the deduced $\dot{E}_{\infty \mathrm{NBI}}$ is the influence of the body's structure on the total energy loss from the orbit, $\dot{E}_{\text {total,BI }} \equiv$ $\dot{E}_{\text {total }}-\dot{E}_{\infty \mathrm{NBI}}$. This measured/deduced body influence on the total energy loss consists of two tiny pieces: the power that actually goes into the body via tidal coupling, $\dot{E}_{\text {body }}$, and the body's tiny influence on the power radiated to infinity, $\dot{E}_{\infty \mathrm{BI}} \equiv \dot{E}_{\infty}-\dot{E}_{\infty \mathrm{NBI}}$ :

$$
\dot{E}_{\text {total,BI }} \equiv \dot{E}_{\infty \mathrm{BI}}+\dot{E}_{\text {body }} .
$$

In principle (as described above), from the observational data plus general-relativity theory we know the body's influence on the total energy loss $\dot{E}_{\text {total,BI }}$ with complete precision. This is not quite what Thorne conjectured, but it is close, and it is the only complete-precision statement we have been able to make about measuring the influence of tidal coupling.

Thorne conjectured we could deduce $\dot{E}_{\text {body }}$ from the observed waves. This in fact appears not to be possible (in principle) with complete precision. However, we argue in Sec. III C and the appendix that, if the central body is highly compact, then the unknown $\dot{E}_{\infty \mathrm{BI}}$ will be smaller than $\dot{E}_{\text {body }}$ by $\sim v^{q} \ll 1$, where $v$ is the orbital velocity and $q$ is some high power; and we show that, when the body's 
external metric is that of Schwarzschild or Kerr, then $q=$ 5. As a result, aside from a very small $\mathrm{O}\left(v^{q}\right)$ uncertainty due to the influence of the body on the energy radiated to infinity, the tidal coupling power $\dot{E}_{\text {body }}$ is equal to the known influence of the body on the total energy loss $\dot{E}_{\text {total, BI}}$.

A brief conclusion is made in Sec. IV.

\section{EXTRACTING THE SPACETIME GEOMETRY AND ORBITAL ELEMENTS}

Aside from allowing tidal coupling, we treat the same class of EMRIs as did Ryan:

First, we assume the central body's exterior spacetime is a vacuum, stationary, axisymmetric, reflection-symmetric, and an asymptotic flat (SARSAF) solution of Einstein's equations. The exterior spacetime metric can be written as (e.g., Eq. (7.1.22) of [21])

$$
d s^{2}=-F(d t-\omega d \phi)^{2}+\frac{1}{F}\left[e^{2 \gamma}\left(d \rho^{2}+d z^{2}\right)+\rho^{2} d \phi^{2}\right],
$$

where $F, \omega$, and $\gamma$ are functions of $\rho$ and $|z|$. In SARSAF spacetimes, there is a one-to-one correspondence between the spacetime metric and a series of scalar multipole moments $\left(M_{2 i}, S_{2 i+1}\right), \quad i=0,1, \cdots[22,23]$. Here $M_{0} \equiv M$ is the mass of the central body, $S_{1}$ is its spin, $M_{2}$ is its mass quadrupole moment, etc. To extract the geometry of the spacetime surrounding the central body, it is sufficient to extract the multipole moments $\left\{M_{\ell}, S_{\ell}\right\}$ [7].

Second, we let a small object with mass $\mu \ll M$ move about the central body in a nearly circular, nearly equatorial orbit.

For precisely circular, equatorial, geodesic motion, the waves have a single fundamental frequency $\Omega_{\phi}$ that is associated with the circular motion $\phi=\Omega_{\phi} t$. When the geodesic orbit is slightly noncircular, it is easy to show that its radius $\rho$ undergoes periodic motion with some angular frequency $\Omega_{\rho}$; and when slightly nonequatorial, its vertical coordinate $z$ undergoes periodic motion with another angular frequency $\Omega_{z}$. These geodesic motions give rise to gravitational waves that are triperiodic: a discrete spectrum with frequencies equal to $m \Omega_{\phi}+k \Omega_{\rho}+n \Omega_{z}$, where $m$, $k$, and $n$ are integers (see [10] for a proof, patterned after the proof by Drasco and Hughes [24] for the Kerr metric). The difference $\Omega_{\rho}-\Omega_{\phi}$ is the frequency of orbital periapsis precession, and $\Omega_{z}-\Omega_{\phi}$ is the frequency of orbital plane precession. These precessions produce corresponding modulations of the gravitational waveforms. (To simplify our prose, we shall refer to $\Omega_{\rho}$ and $\Omega_{z}$ as the "precession frequencies" even though the actual frequencies of precession are $\Omega_{\rho}-\Omega_{\phi}$ and $\Omega_{z}-\Omega_{\phi}$.)

In our case, the orbits are not geodesics; they evolve due to gravitational radiation reaction. But because of the extreme mass ratio, the radiation reaction can be described by the adiabatic approximation, so that on the time scale of an orbital period, the small object moves very nearly along a geodesic of the central body's gravitational field. On a time scale much larger than the orbital period, the object's selfforce causes it to move from one geodesic to another as it loses energy and angular momentum to gravitational radiation. (Note that in this paper, we define the adiabatic approximation to mean that only the dissipative part of the self-force is used to determine the small object's motion; the conservative self-force, which does not cause a secular change in the particle's energy or angular momentum, is entirely neglected. ${ }^{3}$ ) It follows that the three frequencies $\left\{\Omega_{\phi}(t), \Omega_{\rho}(t), \Omega_{z}(t)\right\}$ each evolve with time on the radiation reaction time scale, which is much longer than the orbital periods.

In principle, a large amount of information can be encoded in the time evolution of the waves' three fundamental frequencies $\left\{\Omega_{\phi}(t), \Omega_{\rho}(t), \Omega_{z}(t)\right\}$ and the complex amplitudes (amplitudes and phases) of the various spectral components of the wave amplitude. In terms of the dimensionless gravitational-wave fields $h_{+}$and $h_{\times}$, the spectral components $h_{m \Omega_{\phi}+k \Omega_{\rho}+n \Omega_{z}}$ are as follows (for proof, see [24] for when the central body is a Kerr black hole and [10] for generic central bodies):

$$
h_{+}-i h_{\times}=\sum_{m, k, n} h_{m \Omega_{\phi}+k \Omega_{\rho}+n \Omega_{z}} e^{-i\left(m \Omega_{\phi}+k \Omega_{\rho}+n \Omega_{z}\right) t} .
$$

Thorne's conjecture can be expressed mathematically as the claim that these time-evolving frequencies and amplitudes encode fully and separably,

(1) The values of all the central body's multipole moments $\left\{M_{\ell}, S_{\ell}\right\}$,

(2) The rates $\dot{E}_{\text {body }}$ and $\dot{L}_{\text {body }}$ at which the orbiting object's tidal pull deposits energy and angular momentum into the central body, and

(3) The time-evolving orbital elements, i.e., the orbit's semi-latus rectum $p(t)$, eccentricity $e(t)$, and inclination angle $\iota(t)$.

Ryan's theorem [7] states that, if there is no tidal coupling, then all the SARSAF moments $\left\{M_{2 i}, S_{2 i+1}\right\}$ are encoded in the time-evolving frequencies fully, separably, and redundantly. Ryan did not explicitly address the encoding of the three orbital elements $p(t), e(t)$, and $\iota(t)$.

\footnotetext{
${ }^{3}$ We have neglected the conservative self-force, even though its effects could accumulate over time and affect the phasing of the gravitational waves. For general eccentric nonequatorial orbits, neglecting the conservative self-force might lead to serious errors in the phase of the gravitational waves [25] and thus greatly hinder the recovery of the EMRI's spacetime geometry, orbital parameters, and tidal coupling. However, these errors are reduced by at least the mass ratio $\sim \mu / M$, which we take to be arbitrarily small in this paper. For finite mass ratios such as will be encountered in LIGO and LISA, these errors may be important. For further discussion of the conservative selfforce and its implications for EMRIs, see Refs. [26-28].
} 
However, their encoding is an almost trivial extension of his analysis.

Specifically, Ryan noticed that the three fundamental frequencies are independent of $e$ and $\iota$ to first order in these small quantities, i.e., they are functions solely of the moments and the semi-latus rectum $p$. One can eliminate $p$ by regarding the precession frequencies $\Omega_{z}$ and $\Omega_{\rho}$ as functions of the moments and $\Omega_{\phi}$, or equivalently as functions of the moments and the post-Newtonian (PN) expansion parameter $v \equiv\left(M \Omega_{\phi}\right)^{1 / 3} \simeq$ (orbital velocity). Expanding $\Omega_{z}\left(v ; S_{\ell}, M_{\ell}\right)$ and $\Omega_{\rho}\left(v ; S_{\ell}, M_{\ell}\right)$ in powers of $v$, Ryan found the following pattern of coefficients (with each moment first appearing at a different power of $v$ ), from which all the moments can be extracted separably (Eqs. (18)-(19) of [7]):

$\frac{\Omega_{\rho}}{\Omega_{\phi}}=3 v^{2}-4 \frac{S_{1}}{M^{2}} v^{3}+\left(\frac{9}{2}-\frac{3 M_{2}}{2 M^{3}}\right) v^{4}+\cdots$

$\frac{\Omega_{z}}{\Omega_{\phi}}=2 \frac{S_{1}}{M^{2}} v^{3}+\frac{3 M_{2}}{2 M^{3}} v^{4}+\cdots$.

This result leads to Ryan's algorithm for extracting information. First, from the waves' observed time-evolving precession frequencies and time-evolving primary frequency, one can deduce the functions $\Omega_{z, \rho}\left(\Omega_{\phi}\right)$ and then $\Omega_{z, \rho}(v)$; second, expanding in powers of $v$, one can then read out the multipole moments $\left\{M_{\ell}, S_{\ell}\right\}$ from either $\Omega_{z}(v)$ or $\Omega_{\rho}(v)$.

We almost trivially augment onto Ryan's algorithm the following steps for extracting the time-evolving orbital elements: Third, knowing the moments and then the metric, one can use the geodesic equation to deduce $p(t)$ from $\Omega_{\phi}(t)$. Fourth, one can use wave-generation theory and knowledge of the metric to deduce $e(t)$ and $\iota(t)$ from those amplitudes $h_{m \Omega_{\phi}+k \Omega_{\rho}+n \Omega_{z}}$ of the wave modulations which depend on periapse precession and orbital plane precession. (In practice, only some of the amplitudes $h_{m \Omega_{\phi}+k \Omega_{\rho}+n \Omega_{z}}$ will be large enough to be measurable; Drasco [29] is investigating the relative strength of each $(m, k, n)$ mode when the central body is a Kerr black hole.)

\section{PROBING TIDAL COUPLING}

We now drop Ryan's restriction of no tidal coupling. This does not alter Eqs. (4) for $\Omega_{\rho}$ and $\Omega_{z}$ as functions of $v$, i.e., of the orbital frequency $\Omega_{\phi}$, since all three frequencies only depend on the geodesic motion and hence only depend on the multipole moments $\left\{M_{\ell}, S_{\ell}\right\}$. On the other hand, the evolution of the frequencies, as functions of time, will depend on the tidal coupling.

More generally, we can divide the physical quantities of our analysis into two categories: (i) "static": those quantities related to the geodesic motion of the orbiting object, and (ii) "dynamic": those quantities related to the inspiral of the object (i.e., to the evolving rate at which the object moves from geodesic to geodesic). All static quantities are independent of tidal coupling and all dynamic quantities depend on it.

This suggests that Ryan's analysis can be extended to include tidal coupling. First, the static quantities can be used to deduce the central body's multipole moments, just as in Ryan's original argument as sketched above. Then, the dynamic quantities, combined with knowledge of the spacetime metric, can be used to extract tidal-coupling information. This extension is discussed in the following subsections.

\section{A. The phase evolution when tidal coupling is neglected}

Following Ryan, we characterize the phase evolution of EMRI waves by the number of primary-frequency cycles of waves per logarithmic frequency interval, as a function of the primary waves' slowly increasing frequency $f=$ $\Omega_{\phi} / \pi$. This quantity can be written as (Eq. (4) of [7])

$$
\Delta N(f) \equiv \frac{f d t}{d \ln f}=\frac{f^{2}}{d f / d t} .
$$

This phase evolution $\Delta N(f)$ can be measured by gravitational-wave detectors with high precision.

If there is no tidal coupling and no other influence of the structure of the central body on the waves, as Ryan assumed, then it is possible to read off the multipole moments (and also the small object's mass ${ }^{4}$ ) from a PN expansion of $\Delta N(f)$ (Eq. (57) of [7]):

$$
\begin{aligned}
\Delta N_{\mathrm{NBI}}= & \frac{5}{96 \pi}\left(\frac{M}{\mu}\right) v^{-5}\left[1+\frac{743}{336} v^{2}-4 \pi|v|^{3}+\frac{113}{12} \frac{S_{1}}{M^{2}} v^{3}+\left(\frac{3058673}{1016064}-\frac{1}{16} \frac{S_{1}^{2}}{M^{4}}+5 \frac{M_{2}}{M^{3}}\right) v^{4}\right. \\
& \left.+\sum_{\ell=4,6, \ldots} \frac{(-1)^{\ell / 2}(4 \ell+2)(\ell+1) ! !\left[M_{\ell}+\mathrm{TNILM}\right] v^{2 \ell}}{3 \ell ! ! M^{\ell+1}}+\sum_{\ell=3,5, \ldots} \frac{(-1)^{(\ell-1) / 2}(8 \ell+20) \ell ! !\left[S_{\ell}+\mathrm{TNILM}\right] v^{2 \ell+1}}{3(\ell-1) ! ! M^{\ell+1}}\right] .
\end{aligned}
$$

\footnotetext{
${ }^{4}$ The mass of the small object can be determined from $\Delta N(f)$ even when there is tidal coupling. The leading-PN-order part of the energy flux (equivalently, the leading-PN-order part of $\Delta N(f)$ ) is independent of tidal coupling. One can thus equate the leading-PNorder parts of $\Delta N(f)$ and $\Delta N_{\mathrm{NBI}}$ [Eq. (6)]. After inserting the mass $M$ (obtained from one of the precession frequencies), one can solve for $\mu$. The precession frequencies, in contrast, are independent of $\mu$ [Eq. (4)].
} 
Here "NBI" stands for no body influence and "TNILM" stands for terms nonlinear in lower moments. [Recall that $v=\left(M \Omega_{\phi}\right)^{1 / 3}=(\pi M f)^{1 / 3}$.] So long as tidal coupling is negligible, then, the spacetime multipole moments can be determined redundantly from either $\Delta N(f)$ [Eq. (6)] or the periapse precession frequency $\Omega_{\rho}\left(\Omega_{\phi}\right)$ or the orbital-plane precession frequency $\Omega_{z}\left(\Omega_{\phi}\right)$ [Eqs. (4)].

\section{B. Tidal coupling and the phase evolution}

When tidal coupling effects are included, the redundancy is broken. The multipole moments $\left\{M_{\ell}, S_{\ell}\right\}$ can still [Eq. (4)] be determined from $\Omega_{\rho, z}\left(\Omega_{\phi}\right)$, while (as the following discussion shows), the tidal coupling can be determined from $\left\{M_{\ell}, S_{\ell}\right\}$ and $\Delta N(f)$.

As a preliminary to discussing this, we explain why it is sufficient, in analyzing tidal coupling, to focus on energy exchange between the orbit, the body and the waves, and ignore angular momentum exchange. Since the body is in a (nearly) circular, geodesic orbit, changes in its orbital energy and angular momentum are related by

$$
\dot{E}_{\text {orbit }}=\Omega_{\phi} \dot{L}_{\text {orbit }},
$$

aside from second-order corrections due to the slight orbital ellipticity and inclination angle. Our entire analysis is restricted to first-order corrections, so those second-order corrections are negligible. Similarly, since the energy and angular momentum radiated to infinity are carried by the primary waves, with angular frequency $\omega=2 \pi f=2 \Omega_{\phi}$ (aside from negligible contributions from the precessions, which are second order in the ellipticity and inclination), each graviton carries an energy $\hbar \omega=2 \hbar \Omega_{\phi}$ and an angular momentum $2 \hbar$ (with this last 2 being the graviton spin). Therefore, the energy and angular momentum radiated to infinity are related by

$$
\dot{E}_{\infty}=\Omega_{\phi} \dot{L}_{\infty} .
$$

Conservation of energy and of angular momentum, together with Eqs. (7a) and (7b), then imply that

$$
\dot{E}_{\text {body }}=\Omega_{\phi} \dot{L}_{\text {body }},
$$

for the energy and angular momentum deposited in the body by tidal coupling. Equation (7) implies that, once we understand, observationally, the energy exchange, an understanding of the angular momentum exchange will follow immediately.

Now turn to the influence of the body's internal structure on the observed energy exchange.

The total rate that energy is lost from the orbit (which then goes to infinity and the body) is related to the phase evolution $\Delta N(f)$ by

$$
\dot{E}_{\text {total }}=-\dot{E}_{\text {orbit }}=-\frac{d E_{\text {orbit }}}{d f} \frac{d f}{d t}=-f^{2} \frac{d E_{\text {orbit }}}{d f} \frac{1}{\Delta N} .
$$

The phase evolution $\Delta N$ and the primary frequency $f$ are known from observation, and, after using the precession frequencies to compute the spacetime metric (Sec. II), it is possible to compute $d E_{\text {orbit }} / d f$ via the geodesic equation. ${ }^{5}$ Thus everything on the right-hand side of Eq. (8) can be determined from observed quantities, which means that $\dot{E}_{\text {total }}$ is measurable.

Another measurable quantity, we claim, is the rate that energy would be lost from the orbit if the body's structure had no influence. This quantity is [by analogy with Eq. (8)]

$$
\dot{E}_{\text {total, } \mathrm{NBI}}=-f^{2} \frac{d E_{\text {orbit }}}{d f} \frac{1}{\Delta N_{\mathrm{NBI}}} .
$$

Knowing the moments as a function of frequency from measurements of the precessions, $\Delta N_{\mathrm{NBI}}$ can be computed from the moments via Ryan's phasing relation ${ }^{6}$ (6), and, as we have seen, $d E_{\text {orbit }} / d f$ can also be computed from the observations; so $\dot{E}_{\text {total,NBI }}$ is, indeed, observable, as claimed. Therefore the influence of the body's structure on the orbit's total energy loss

$$
\dot{E}_{\text {total,BI }}=\dot{E}_{\text {total }}-\dot{E}_{\text {total,NBI }}
$$

is also observable.

This body influence on the total energy loss consists of two parts: the energy that goes into the body via tidal coupling, $\dot{E}_{\text {body }}$, and a tiny body-influenced modification of the rate that the waves carry energy to infinity

$$
\dot{E}_{\text {total,BI }}=\dot{E}_{\text {body }}+\dot{E}_{\infty \mathrm{BI}} \text {, }
$$

where

$$
\dot{E}_{\infty \mathrm{BI}}=\dot{E}_{\infty}-\dot{E}_{\mathrm{total}, \mathrm{NBI}} .
$$

Thorne conjectured that the energy exchange due to tidal coupling, $\dot{E}_{\text {body }}$, would be observable. We doubt very much that it is, since in general we see no way to determine the relative contributions of $\dot{E}_{\text {body }}$ and $\dot{E}_{\infty \mathrm{BI}}$ to the observed total body influence $\dot{E}_{\text {total,BI}}$. The best one can do, in general, in validating Thorne's conjecture, is to extract the central body's total influence on the orbital energy loss, $\dot{E}_{\text {total,BI}}$. However, in the special case of a body that is exceedingly compact, we can do better, as we shall explain in the next subsection.

\footnotetext{
${ }^{5}$ To do this, first insert the multipole moments $\left\{M_{\ell}, S_{\ell}\right\}$ into the geodesic equation. Then, solve the geodesic equation for the family of circular, equatorial orbits about the central body. Each orbit $i$ will have a particular value of energy $E_{\text {orbit, } i}$ and frequency $f_{i}$; this one-to-one mapping between $E_{\text {orbit }}$ and $f$ can then be used to compute $d E_{\text {orbit }} / d f$.

${ }^{6}$ Ryan calculates the phasing relation to $2 \mathrm{PN}$ order (i.e., to $O\left(v^{4}\right)$ past leading order). By extending Ryan's calculation to higher post-Newtonian orders, the terms omitted from Eq. (6) can be written explicitly.
} 


\section{The dependence of the $\dot{E}_{\infty}$ on the central body's internal structure}

Consider a central body sufficiently compact that gravity near its surface blue-shifts the orbiting object's tidal field, making it appear like ingoing gravitational waves as seen by stationary observers. This is the case, for example, when the central body is a black hole. Then, we claim, the ratio $\dot{E}_{\infty \mathrm{BI}} / \dot{E}_{\text {body }}$ is very small:

$$
\frac{\dot{E}_{\infty \mathrm{BI}}}{\dot{E}_{\mathrm{body}}} \sim v^{q} \ll 1,
$$

where $q$ is a large number, very likely 5. For LISA, almost all of the wave cycles used in extracting information from the waves will be from radii where $v \leqq 0.5$ so $v^{5} \lesssim 0.03$. For example, for a Kerr black hole, if the spin parameter is $a / M \lessgtr 0.5$, then at the innermost stable circular orbit, $v \lesssim 0.5$. Consequently, almost all of the measured $\dot{E}_{\text {total,BI }}$ will go into the body itself via tidal coupling, so $\dot{E}_{\text {body }}$ will be measured to good accuracy.

To understand our claim that $\dot{E}_{\infty \mathrm{BI}} / \dot{E}_{\text {body }} \sim v^{q}$ for some large $q$, consider a central body whose external metric is that of a Kerr black hole. In this case, one can use the Teukolsky formalism [30] (first-order perturbation theory in the mass ratio $\mu / M$ ) to compute the energies radiated to infinity and tidally coupled into the central body. We have carried out that Teukolsky analysis for general $a / M$ and present the details for the special case $a=0$ in the appendix. Here we explain the underlying physics. We begin with some preliminaries:

We need only consider the primary-frequency waves, $f=\Omega_{\phi} / \pi$, since they account for all the energy loss and transfer, up to corrections second order in the eccentricity $e$ and inclination angle $\iota$. This means, correspondingly, that we can restrict ourselves to a precisely circular and equatorial orbit. The waves and tidal coupling then have predominantly spheroidal harmonic order $\ell=m=2$ and frequency $f$ (angular frequency $\omega=2 \pi f=2 \Omega_{\phi}$ ). Since we only want to know, to within factors of order unity, the ratio $\dot{E}_{\infty \mathrm{BI}} / \dot{E}_{\text {body }}$, it will be sufficient to restrict ourselves to these dominant $\ell=m=2, \omega=2 \Omega_{\phi}$ perturbations.

In the Teukolsky formalism, these perturbations are embodied in a radial "wave function" that can be normalized in a variety of different ways. The usual normalization, based on the Newman-Penrose field $\psi_{4}$, is bad for physical insight because it treats outgoing waves and ingoing waves quite differently (see the appendix). One normalization that treats them on the same footing sets the radial wave function equal to that of the tidal gravitational field ("electric-type" components of the Weyl or Riemann curvature tensor) measured by "zero-angularmomentum" observers, ZAMOs (a family of observers, each of whom resides at fixed radius $r$ and polar angle $\theta$ ). We shall denote that tidal field [with $e^{-i \omega t} \times$ (spheroidal harmonic) factored out so the field is complex, not real] by $\mathcal{E}$. Another, closely related normalization for the radial wave function sets its modulus squared equal to the rate of flow of energy. We shall denote this choice by $\Psi$. At large radii, $\mathcal{E} \sim\left(\ddot{h}_{+}+i \ddot{h}_{\times}\right)=\omega^{2}\left(h_{+}+i h_{\times}\right)$, where $h_{+}$and $h_{\times}$are the dimensionless gravitational wave fields; so the radiated energy is $\dot{E}_{\infty} \sim r^{2} \mid \dot{h}_{+}+$ $\left.i \dot{h}_{\times}\right|^{2} \sim(r / \omega)^{2} \mathcal{E}_{\infty}^{2}$, which tells us that $\Psi_{\infty} \sim(r / \omega) \mathcal{E}_{\infty}$. Near the body's surface (i.e., near where the horizon would be if the body were a Kerr black hole), the energy flux is $\dot{E} \sim(r / \omega)^{2}\left|\alpha^{2} \mathcal{E}\right|^{2}$, where $\alpha$ is the Kerr-metric lapse function, which goes to zero at the horizon radius. (The ZAMOs' divergently large outward speed, relative to infalling observers, causes them to see a divergently large tidal field; the factor $\alpha^{2}$ corrects for that divergence; see, e.g., the discussion in Sec. VI.C.2 of [31].) Thus, in order to ensure that the power flow is the square of the renormalized radial wave function,

$$
\dot{E} \sim|\Psi|^{2},
$$

we must renormalize the ZAMO-measured tidal field $\mathcal{E}$ by $\Psi \sim(r / \omega) \mathcal{E} \quad$ at $r \rightarrow \infty, \quad \Psi \sim\left(\alpha^{2} r / \omega\right) \mathcal{E}$ near body.

With these preliminaries finished, we can give our physical argument for Eq. (13) in terms of the radial wave function $\Psi$. Our argument relies on Fig. 1.

If the central body is a Kerr black hole, then the boundary condition on $\Psi$ at its surface (the horizon) is purely downgoing waves, and at infinity, purely outgoing waves. The ratio of downgoing power at the horizon to outgoing power at infinity has the standard Kerr values [32,33]: $\dot{E}_{\text {body }} / \dot{E}_{\infty \mathrm{NBI}} \sim \boldsymbol{v}^{8}$ if the hole's spin angular velocity $\Omega_{H}$ is much less than the orbital angular velocity $\Omega_{\phi}$; and $\dot{E}_{\text {body }} / \dot{E}_{\infty \mathrm{NBI}} \sim v^{5}$ if $\Omega_{H} \gg \Omega_{\phi}$. (Here we have used the no-body-influence notation $\dot{E}_{\infty \mathrm{NBI}}$ for the outgoing power because a central black hole's internal structure is unable to influence the waves radiated to infinity.) Correspondingly, by virtue of Eq. (14), the ratio of the downgoing field at the horizon $\Psi_{\downarrow}$ to the outgoing field at infinity $\Psi_{\infty \mathrm{NBI}}$ is

$$
\frac{\Psi_{\downarrow}}{\Psi_{\infty \mathrm{NBI}}} \sim\left\{\begin{array}{c}
v^{4} \\
v^{5 / 2}
\end{array}\right\} \text { for }\left\{\begin{array}{l}
\Omega_{H} \ll \Omega_{\phi} \\
\Omega_{H} \gg \Omega_{\phi}
\end{array}\right\} .
$$

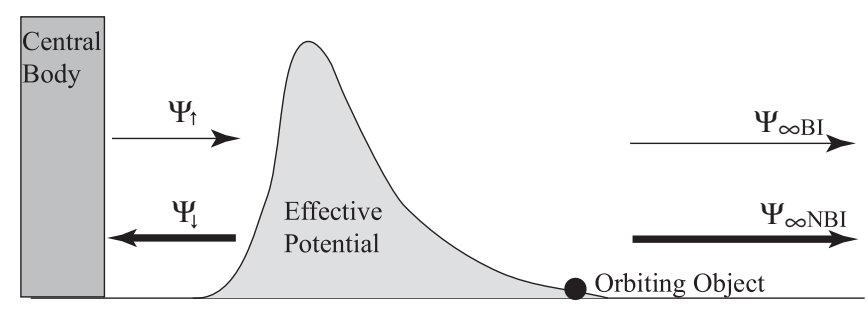

FIG. 1. The renormalized tidal gravitational fields $\Psi$ produced near a central body's surface and at large radii by the orbiting object, when the central body has the same exterior metric as a Kerr black hole. 
This suppression of the downgoing field relative to the outgoing is due, mathematically, to a reflective effective potential in the wave equation that $\Psi$ satisfies (Fig. 1). Physically, it is due to coupling of the field $\Psi$ to the central body's spacetime curvature.

Now suppose the central body is not a black hole, but some other object so compact that its surface is well beneath the peak of the effective potential. This mathematical assumption is equivalent to our physical assumption that the ZAMOs see the downgoing field $\Psi_{\downarrow}$ so strongly blue-shifted by the central body's gravity that it looks like radiation. The only way, then, that the central body can influence the energy radiated to infinity is to reflect a portion of this downgoing radiation back upward. Mathematically, this corresponds to replacing the black hole's downgoing boundary condition by

$$
\Psi_{\uparrow}=\mathcal{R} \psi_{\downarrow}
$$

at some chosen radius just above the body's surface. Here $\Psi_{\downarrow}$ and $\Psi_{\uparrow}$ are the downgoing and upgoing components of $\Psi$; see Fig. 1. For simplicity, we shall assume that the amplitude reflection coefficient $\mathcal{R}$ is small, $|\mathcal{R}| \ll 1$. Otherwise we would have to deal with a possible resonant buildup of energy between the reflective central body and the reflective effective potential-though that would not change our final answer (see, e.g., the more detailed analysis in the appendix).

The upgoing waves $\Psi_{\uparrow}$ have great difficulty getting through the effective potential. The fraction of the upgoing power that gets transmitted through, successfully, is $\sim(M \omega)^{6}$ if the hole rotates slowly, and $\sim(M \omega)^{5}$ if rapidly [Eq. (8.83) of [31] with $\ell=2$ and $\sigma_{\infty}=\omega$ ]. Since the fields $\Psi$ are the square roots of the powers (aside from complex phase) and since $M \omega=2 M \Omega_{\phi}=2 v^{3}$, this power transmissivity corresponds to

$$
\frac{\Psi_{\infty \mathrm{BI}}}{\Psi_{\uparrow}} \sim\left\{\begin{array}{c}
v^{9} \\
v^{15 / 2}
\end{array}\right\} \quad \text { for }\left\{\begin{array}{l}
\Omega_{H} \ll \Omega_{\phi} \\
\Omega_{H} \gg \Omega_{\phi}
\end{array}\right\} .
$$

Combining Eqs. (16)-(18), we see that

$$
\frac{\Psi_{\infty \mathrm{BI}}}{\Psi_{\infty \mathrm{NBI}}} \sim\left\{\begin{array}{l}
v^{13} \\
v^{10}
\end{array}\right\} \text { for }\left\{\begin{array}{l}
\Omega_{H} \ll \Omega_{\phi} \\
\Omega_{H} \gg \Omega_{\phi}
\end{array}\right\} .
$$

If these two complex outgoing fields are not precisely out of phase with each other (phase difference $\pm \pi / 2$ ), then the outgoing power is $\left|\Psi_{\infty \mathrm{NBI}}+\Psi_{\infty \mathrm{BI}}\right|^{2} \simeq\left|\Psi_{\infty \mathrm{NBI}}\right|^{2}+$ $2 \mathfrak{R}\left(\Psi_{\infty \mathrm{NBI}} \Psi_{\infty \mathrm{BI}}^{*}\right)$, which means that the ratio of the radiated body-influenced power to radiated no-body-influence power is

$$
\frac{\dot{E}_{\infty \mathrm{BI}}}{\dot{E}_{\infty \mathrm{NBI}}} \sim\left\{\begin{array}{c}
v^{13} \\
v^{10}
\end{array}\right\} \text { for }\left\{\begin{array}{l}
\Omega_{H} \ll \Omega_{\phi} \\
\Omega_{H} \gg \Omega_{\phi}
\end{array}\right\}
$$

In the unlikely case (which we shall ignore) that the two fields are precisely out of phase, the ratio will be the square of this.

By combining Eq. (20) with the square of Eq. (16), we obtain the ratio of the body-influence power radiated to infinity over the tidal coupling power into the central body:

$$
\dot{E}_{\infty \mathrm{BI}} / \dot{E}_{\text {body }} \sim v^{5}
$$

independent of whether the body rotates slowly or rapidly. This is the claimed result.

If the central body's external metric is not Kerr, then the first-order perturbation equations for the orbiting body's spacetime curvature will probably not be separable in $\{r, \theta\}$, so the analysis will be much more complex. Nevertheless the physical situation presumably is unchanged in this sense: The body's spacetime curvature will couple to the perturbation field in such a way as to resist energy flow through the region between the body's surface and the object's orbit. Correspondingly, the perturbation fields and power flows are very likely to behave in the same manner as for the Kerr metric, with the same final result, $\dot{E}_{\text {body }} / \dot{E}_{\infty \mathrm{BI}} \sim v^{q}$ with $q$ very likely still 5 but possibly some other number significantly larger than 1 .

If this is, indeed, the case, then for any sufficiently compact central body the power tidally deposited into the body $\dot{E}_{\text {body }}$ will be very nearly equal to $\dot{E}_{\text {total,BI}}$, which is measurable; and therefore the tidal power will be measurable.

\section{CONCLUSION}

In this paper, we have extended Ryan's analysis to show that in principle it is possible to recover not only the spacetime geometry of the central body, but also the evolving orbital parameters of the inspiraling object and the evolving tidal coupling between the small object and the central body. Therefore, in principle we can obtain a full description of the SARSAF spacetime, the tidal coupling, and the inspiral orbit from EMRI or IMRI waveforms. In practice, the method of extracting the information is likely to be quite different from the algorithm we have presented here.

Further generalizations of Ryan's theorem and development of practical methods to implement it are topics of our ongoing research.

\section{ACKNOWLEDGMENTS}

We would like to thank Yanbei Chen, Steve Drasco, Yi Pan, and Kip Thorne for helpful discussions, Drasco for carefully reading a draft of this paper, and Thorne for assistance with the prose of this paper. This work was supported in part by NSF grants PHY-0099568 and PHY0601459, NASA grants NAG5-12834 and NNG04GK98G, and the Brinson Foundation. 


\section{APPENDIX A: AN EXPLICIT DERIVATION OF RESULTS IN SECTION III C}

\section{Teukolsky perturbation formalism}

In this subsection, we use the Teukolsky perturbation theory to justify our results in Sec. III C. We first briefly review the standard Teukolsky perturbation formalism. Details can be found, e.g., in [34]. To shorten our expressions, in this appendix we restrict ourselves to a nonrotating central body with external metric the same as a Schwarzschild black hole but with a finite reflectivity. The generalization to the Kerr metric is straightforward but with more cumbersome algebra. We have carried it out, obtaining the same result as is found by the physical argument in the text.

In the Teukolsky formalism, people usually calculate the perturbation to a Newman-Penrose quantity $\psi_{4}$ that is related to the ZAMO-measured tidal field $\mathcal{E}$ by a linear transformation of the basis vectors. This $\psi_{4}$ can be decomposed into Fourier-Harmonic components according to

$$
\psi_{4}=\frac{1}{r^{4}} \int_{-\infty}^{\infty} d \omega \sum_{l m} R_{\omega l m}(r)_{-2} Y_{l m}(\theta, \phi) e^{-i \omega t},
$$

where $-2 Y_{l m}(\theta, \phi)$ are the spin-weighted spherical harmonics. The radial function $R_{\omega l m(r)}$ satisfies the inhomogeneous Teukolsky equation

$$
\left[r^{2} \alpha^{2} \frac{d^{2}}{d r^{2}}-2(r-M) \frac{d}{d r}+U(r)\right] R_{\omega l m}(r)=T_{\omega l m},
$$

where $\alpha^{2}=1-2 M / r$ is the lapse function for the Schwarzschild metric. The expressions for the potential $U(r)$ and the source $T_{\omega l m}$ can be found, e.g., in [32], Eqs. (2.3), (A1).

In order to solve this equation, we construct two linearly independent solutions to the homogeneous Teukolsky equation, which satisfy the following boundary conditions,

$$
\begin{aligned}
& R_{\omega l m}^{\mathrm{IN}} \rightarrow \begin{cases}(\omega r)^{4} \alpha^{4} e^{-i \omega r^{*}}, & r \rightarrow 2 M \\
(\omega r)^{-1} Q_{\omega l m}^{\text {in }} e^{-i \omega r^{*}}+(\omega r)^{3} Q_{\omega l m}^{\text {out }} e^{i \omega r^{*}}, & r \rightarrow+\infty\end{cases} \\
& R_{\omega l m}^{\mathrm{UP}} \rightarrow \begin{cases}(\omega r)^{4} \alpha^{4} P_{\omega l m}^{\text {out }} e^{-i \omega r^{*}}+P_{\omega l m}^{\text {in }} e^{i \omega r^{*}}, & r \rightarrow 2 M \\
(\omega r)^{3} e^{i \omega r^{*}}, & r \rightarrow+\infty\end{cases}
\end{aligned}
$$

where $d / d r^{*}=\alpha^{2} d / d r$. From these two homogeneous solutions, we can construct the inhomogeneous solution according to

$$
\begin{aligned}
R_{\omega l m}(r)= & \frac{1}{\text { Wronskian }\left[R_{\omega l m}^{\mathrm{UP}}, R_{\omega l m}^{\mathrm{IN}}\right]} \\
& \times\left(R_{\omega l m}^{\mathrm{UP}}(r) \int_{2 M}^{r} d r^{\prime} R_{\omega l m}^{\mathrm{IN}}\left(r^{\prime}\right) \mathcal{T}_{\omega l m}\left(r^{\prime}\right)\right. \\
& \left.+R_{\omega l m}^{\mathrm{IN}}(r) \int_{r}^{\infty} d r^{\prime} R_{\omega l m}^{\mathrm{UP}}\left(r^{\prime}\right) \mathcal{T}_{\omega l m}\left(r^{\prime}\right)\right),
\end{aligned}
$$

where $\mathcal{T}_{\omega l m}(r) \equiv T_{\omega l m}(r)\left(r^{2}-2 M r\right)^{-2}$. This solution has only outgoing waves at infinity and satisfies the purely ingoing boundary condition: ([32], Eqs. (2.8) and (2.9))

$$
\begin{aligned}
R_{\omega l m}(r \rightarrow \infty) & \sim \mu \omega^{2} Z_{\omega l m}^{\mathrm{IN}} r^{3} e^{i \omega r^{*}}, \\
R_{\omega l m}(r \rightarrow 2 M) & \sim \mu \omega^{3} Z_{\omega l m}^{\mathrm{UP}} r^{4} \alpha^{4} e^{-i \omega r^{*}},
\end{aligned}
$$

where

$$
\begin{aligned}
Z_{\omega l m}^{\mathrm{IN}, \mathrm{UP}}= & \frac{1}{2 i \mu \omega^{2} Q_{\omega l m}^{\mathrm{in}}} \\
& \times \int_{2 M}^{\infty} d r\left[\left(r^{2}-2 M r\right)^{-2} R_{\omega l m}^{\mathrm{IN}, \mathrm{UP}}(r) T_{\omega l m}(r)\right] .
\end{aligned}
$$

At infinity, where the spacetime is almost flat, $\psi_{4}$ is directly related to the outgoing gravitational wave strains according to

$$
\psi_{4}=\frac{1}{2}\left(\ddot{h}_{+}-i \ddot{h}_{\times}\right),
$$

and we can obtain the luminosity formula ([32], Eq. (2.21))

$$
\dot{E}_{\infty}=\frac{1}{4 \pi}\left(\frac{\mu}{M}\right)^{2} \sum_{l m}(M \omega)^{2}\left|Z_{\omega l m}^{\mathrm{IN}}\right|^{2} .
$$

\section{Inner boundary condition}

The above purely ingoing boundary condition makes sense when the central body is a black hole because we know everything is absorbed at the horizon of the black hole. If the central body is some other kind of object, the only way it can influence the perturbation field $R_{\omega l m}$ just above its surface is by producing an outgoing-wave component via some effective reflectivity $\mathcal{R}$. The result will be a modified field

$$
R_{\omega l m}(r \rightarrow 2 M) \sim e^{-i \omega r^{*}}+\text { (something) } e^{i \omega r^{*}} .
$$

The "something" will be proportional to $\mathcal{R}$, and it will also have a peculiar radial dependence, because $\psi_{4}$ relies for its definition on an ingoing null tetrad and thereby treats ingoing and outgoing waves in very different manners.

\section{Chandrasekhar transform}

To learn what the something should be, we can transform to a new radial wave function that treats ingoing and outgoing waves on the same footing. Two such functions were introduced and used in Sec. IIIC: the ZAMOmeasured tidal field $\mathcal{E}$ and a field $\Psi$ whose modulus squared is the power flow, for both outgoing and ingoing waves. Those choices are good for Sec. III C's physical, order-of-magnitude arguments, but at general radii $r$ they not related in any simple way to $\psi_{4}$. A choice that is simply related to $\psi_{4}$ is the Regge-Wheeler function $X$, and we shall use it here.

The radial wave function $R$ for the Newman-Penrose $\psi_{4}$ is related to the Regge-Wheeler function $X$ by the 
Chandrasekhar transform, Eq. (A6) of [32]. This Chandrasekhar transform takes the form

$$
R_{\omega l m}^{\mathrm{IN}, \mathrm{UP}}=\chi_{\omega l m}^{\mathrm{IN}, \mathrm{UP}} C_{\omega} X_{\omega l m}^{\mathrm{IN}, \mathrm{UP}},
$$

where

$\chi_{\omega l m}^{\mathrm{IN}}=\frac{16(1-2 i M \omega)(1-4 i M \omega)(1+4 i M \omega)}{(l-1) l(l+1)(l+2)-12 i M \omega}(M \omega)^{3}$,

$\chi_{\omega l m}^{\mathrm{UP}}=-\frac{1}{4}$.

$C_{\omega}$ is a second-order differential operator, and $X_{\omega l m}^{\mathrm{IN}, \mathrm{UP}}$ are two linearly independent solutions of the homogeneous Regge-Wheeler equation

$$
\left[\frac{d^{2}}{d r^{* 2}}+\omega^{2}-V(r)\right] X_{\omega l m}(r)=0,
$$

where

$$
V(r)=\alpha^{2}\left[\frac{l(l-1)}{r^{2}}-\frac{6 M}{r^{3}}\right]
$$

The asymptotic expressions for $X_{\omega l m}^{\mathrm{IN}, \mathrm{UP}}$ are ([32], Eq. (2.7))

$$
\begin{gathered}
X_{\omega l m}^{\mathrm{IN}} \rightarrow \begin{cases}e^{-i \omega r^{*}}, & r \rightarrow 2 M \\
A_{\omega l m}^{\text {in }} e^{-i \omega r^{*}}+A_{\omega l m}^{\text {out }} e^{i \omega r^{*}}, & r \rightarrow+\infty\end{cases} \\
X_{\omega l m}^{\mathrm{UP}} \rightarrow \begin{cases}-B_{\omega l m}^{\text {out }} e^{-i \omega r^{*}}+B_{\omega l m}^{\text {in }} e^{i \omega r^{*}}, & r \rightarrow 2 M \\
e^{i \omega r^{*}}, & r \rightarrow+\infty .\end{cases}
\end{gathered}
$$

Here we note that by the conservation of the Wronskian, it is straightforward to show that $B^{\text {in,out }}=A^{\text {in,out }}$.

\section{4. $\dot{\boldsymbol{E}}_{\infty}$ with a reflective inner boundary condition}

Because the Regge-Wheeler function treats outgoing and ingoing waves on the same footing, the desired, reflective inner boundary condition for it takes the simple form

$$
\tilde{X}_{\omega l m}^{\mathrm{IN}}(r \rightarrow 2 M) \sim e^{-i \omega r^{*}}+\mathcal{R} e^{i \omega r^{*}} .
$$

Here $\tilde{X}_{\omega l m}^{\mathrm{IN}}$ is a new homogeneous solution of the ReggeWheeler equation.

This new homogeneous solution is a superposition of both ingoing and outgoing waves at the horizon. It is shown in [35] that because the Regge-Wheeler function treats outgoing and ingoing waves in the same manner, $|\mathcal{R}|^{2}$ has the physical meaning of the energy flux reflectivity, i.e., the ratio between outgoing and ingoing energy flux at the horizon.
The homogeneous solution (A15) which satisfies the new inner boundary condition can be constructed from the old homogeneous solutions:

$$
\tilde{X}_{\omega l m}^{\mathrm{IN}}=\beta_{1} X_{\omega l m}^{\mathrm{IN}}+\beta_{2} X_{\omega l m}^{\mathrm{UP}},
$$

where

$$
\beta_{1}=1+\frac{\mathcal{R} A_{\omega l m}^{\text {out }}}{A_{\omega l m}^{\text {in }}}, \quad \beta_{2}=\frac{\mathcal{R}}{A_{\omega l m}^{\text {in }}} .
$$

After doing an inverse Chandrasekhar transform, we obtain the corresponding homogeneous solution of the homogeneous Teukolsky equation

$$
\tilde{R}_{\omega l m}^{\mathrm{IN}}=R_{\omega l m}^{\mathrm{IN}}+\frac{\beta_{2}}{\beta_{1}} \frac{\chi_{\omega l m}^{\mathrm{IN}}}{\chi_{\omega l m}^{\mathrm{UP}}} R_{\omega l m}^{\mathrm{UP}} .
$$

Now we can replace $R^{\mathrm{IN}}$ by $\tilde{R}^{\mathrm{IN}}$ in Eq. (A4) to obtain the solution $\tilde{R}_{\omega l m}(r)$ which satisfies the inhomogeneous Teukolsky equation with upgoing and downgoing waves at the horizon and purely outgoing waves at infinity. From this $\tilde{R}_{\omega l m}(r)$ we identify the new amplitudes $\tilde{Z}_{\omega l m}^{\mathrm{IN}}$ as in Eq. (A5):

$$
\tilde{Z}_{\omega l m}^{\mathrm{IN}}=Z_{\omega l m}^{\mathrm{IN}}+\frac{\beta_{2}}{\beta_{1}} \frac{\chi_{\omega l m}^{\mathrm{IN}}}{\chi_{\omega l m}^{\mathrm{IP}}} Z_{\omega l m}^{\mathrm{UP}}
$$

From these new $\tilde{Z}_{\omega l m}^{\mathrm{IN}}$ the calculation of the luminosity at infinity is straightforward.

In [32] Poisson and Sasaki have already worked out all the relevant formulae, so we only give the results. For the original expressions in [32], please refer to Eq. (3.25) for $A^{\text {in }}, A^{\text {out}}$; Eq. (A7) for $\chi^{\mathrm{IN}}, \chi^{\mathrm{UP}}$; and Eqs. (5.4), (5.6), (5.11), (5.12) for $Z_{\omega l m}^{\mathrm{IN}}, Z_{\omega l m}^{\mathrm{UP}}$.

The leading luminosity correction comes from the $l=$ $2, m= \pm 2$ mode, and we have

$$
\dot{E}_{\infty}=\left.\dot{E}_{\infty}\right|_{\text {Schwarzschild }}\left|1-\frac{128 i \mathcal{R} v^{13}}{15 \beta_{1}}\right|^{2},
$$

where $v$ is the same PN expansion parameter as that in Sec. III C. Unless the reflection coefficient $\mathcal{R}$ is precisely real, this gives

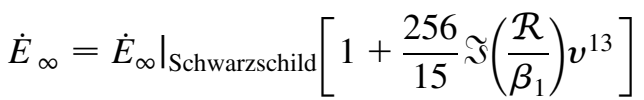

in agreement with Eq. (20). The change in $\dot{E}_{\text {body }}$ should be

$$
\dot{E}_{\text {body }}=\left.\dot{E}_{\text {body }}\right|_{\text {Schwarzschild }}\left(\frac{1-|\mathcal{R}|^{2}}{\left|\beta_{1}\right|^{2}}\right)
$$

where $\beta_{1}$ is defined in Eq. (A17). 
[1] M. Colpi, S. L. Shapiro, and I. Wasserman, Phys. Rev. Lett. 57, 2485 (1986).

[2] F. D. Ryan, Phys. Rev. D 55, 6081 (1997).

[3] T. D. Lee, Phys. Rev. D 35, 3637 (1987).

[4] D. Brown et al., Phys. Rev. Lett. 99, 201102 (2007).

[5] I. Mandel, D. Brown, J. Gair, and C. Miller, arXiv:0705.0285.

[6] A. Abramovici et al., Science 256, 325 (1992).

[7] F. D. Ryan, Phys. Rev. D 52, 5707 (1995).

[8] K.S. Thorne, in The Future of Theoretical Physics and Cosmology: Celebrating Stephen Hawking's 60th Birthday, edited by G.W. Gibbons, S. J. Rankin, and E.P.S. Shellard (Cambridge University Press, Cambridge, England, 2003), pp. 74-104.

[9] T. Prince and K. Danzmann, Tech. Rep., LISA International Science Team (2005), http://www.srl.caltech. edu/lisa/documents/SRD_v3_0_sb.pdf.

[10] C. Li (unpublished).

[11] J. R. Gair et al., Classical Quantum Gravity 21, S1595 (2004).

[12] J. Gair and L. Wen, Classical Quantum Gravity 22, S1359 (2005).

[13] D. Brown (unpublished).

[14] N. A. Collins and S. A. Hughes, Phys. Rev. D 69, 124022 (2004).

[15] K. Glampedakis and S. Babak, Classical Quantum Gravity 23, 4167 (2006).

[16] A. Stroeer, J. Gair, and A. Vecchio, in Laser Interferometer Space Antenna: 6th International LISA Symposium, edited by S. Merkowitz and J. C. Livas, AIP Conference Proceedings No. 873 (AIP, New York, 2006), p. 444.

[17] T.P. Sotiriou and T.A. Apostolatos, Phys. Rev. D 71, 044005 (2005).
[18] H. Fang and G. Lovelace, Phys. Rev. D 72, 124016 (2005).

[19] Y. Rathore, R. Blandford, and A. Broderick, Mon. Not. R. Astron. Soc. 357, 834 (2005).

[20] D. Lai and Y. Wu, Phys. Rev. D 74, 024007 (2006).

[21] R. M. Wald, General Relativity (University of Chicago Press, Chicago, 1984).

[22] R. Geroch, J. Math. Phys. (N.Y.) 11, 2580 (1970).

[23] R. Hansen, J. Math. Phys. (N.Y.) 15, 46 (1974).

[24] S. Drasco and S. A. Hughes, Phys. Rev. D 73, 024027 (2006).

[25] A. Pound, E. Poisson, and B. G. Nickel, Phys. Rev. D 72, 124001 (2005).

[26] A. Pound and E. Poisson, Phys. Rev. D 77, 044013 (2008).

[27] A. Pound and E. Poisson, Phys. Rev. D 77, 044012 (2008).

[28] E. Poisson, Living Rev. Relativity 7, 6 (2004), http:// relativity.livingreviews.org/Articles/lrr-2004-6/ (cited on 28 Feb. 2007)

[29] S. Drasco, arXiv:0711.4644.

[30] S. A. Teukolsky, Astrophys. J. 185, 635 (1973).

[31] K. S. Thorne, R. Price, and D. MacDonald, Black Holes: The Membrane Paradigm (Yale University Press, New Haven, 1986).

[32] E. Poisson and M. Sasaki, Phys. Rev. D 51, 5753 (1995).

[33] H. Tagoshi, S. Mano, and E. Takasugi, Prog. Theor. Phys. 98, 829 (1997).

[34] M. Sasaki and H. Tagoshi, Living Rev. Relativity 6, 6 (2003), http://www.livingreviews.org/lrr-2003-6 (cited on 28 Feb. 2007).

[35] S. Chandrasekhar, The Mathematical Theory of Black Holes (Oxford University Press, Oxford, 1985), Ch. 4, 9. 